\title{
Mitochondrial aldehyde dehydrogenase activity protects against lipopolysaccharide-induced cardiac dysfunction in rats
}

\author{
YING HU ${ }^{1}$, JIN-BIN YAN ${ }^{2}$, MING-ZHI ZHENG ${ }^{3}$, XING-HUI SONG ${ }^{2}$, \\ LIN-LIN WANG ${ }^{2}$, YUE-LIANG SHEN ${ }^{2}$ and YING-YING $\mathrm{CHEN}^{2}$ \\ ${ }^{1}$ Department of Ultrasonography, The First Affiliated Hospital, College of Medicine, Zhejiang University, Hangzhou, \\ Zhejiang 310003; ${ }^{2}$ Department of Basic Medical Sciences, Zhejiang University School of Medicine, Hangzhou, \\ Zhejiang 310058; ${ }^{3}$ Department of Pharmacology, Zhejiang Medical College, Hangzhou, Zhejiang 310053, P.R. China
}

Received December 31, 2013; Accepted September 18, 2014

DOI: $10.3892 / \mathrm{mmr} .2014 .2803$

\begin{abstract}
Myocardial dysfunction in sepsis is associated with an increased risk of mortality. The mitochondrial aldehyde dehydrogenase (ALDH2) enzyme is crucial for protecting the heart from ischemic injury. The aim of the present study was to determine the role of ALDH2 in cardiac dysfunction induced by lipopolysaccharide (LPS). Male rats were treated intraperitoneally with LPS, and their stroke volume and cardiac output were evaluated using an M-mode echocardiograph. The expression levels and activity of ALDH2, nitric oxide content and inducible nitric oxide synthase (iNOS) activity, and the opening of the mitochondrial permeability transition pore (MPTP) were also evaluated. Treatment with LPS $(5,10$, or $20 \mathrm{mg} / \mathrm{kg}$ ) resulted in a steady decrease in cardiac output and stroke volume. The ALDH2 activity was decreased in a dose-dependent manner; however, the ALDH 2 protein expression levels remained unchanged. Alda-1, a specific activator of ALDH2, increased the activity of ALDH2 and lessened LPS-induced cardiac dysfunction. A marked opening of the MPTP was observed $12 \mathrm{~h}$ following treatment with LPS, which was prevented by Alda- 1 . The improvement in cardiac function in response to treatment with Alda-1, was partially prevented by treatment with the MPTP inhibitor atractyloside. LPS treatment induced an increase in iNOS activation and inhibition of ALDH2 activity. The iNOS selective inhibitor, aminoguanidine, partially reversed the LPS-induced ALDH2 activity decrease and MPTP opening. These results indicate that ALDH2 activity may have a role in protecting against LPS-induced cardiac dysfunction. The potential mechanism may involve inhibition of MPTP opening and iNOS expression.
\end{abstract}

Correspondence to: Dr Ying-Ying Chen, Department of Basic Medical Sciences, Zhejiang University School of Medicine, 866 Yu-Hang-Tang Road, Hangzhou 310058, P.R. China

E-mail: bchenyy@zju.edu.cn

Key words: sepsis, lipopolysaccharide, aldehyde dehydrogenase, mitochondria

\section{Introduction}

Sepsis is a deleterious host response to infection that leads to septic shock. It is a major health care problem affecting millions of people each year. Sepsis is associated with a high mortality rate, and despite the adoption of guideline recommendations, sepsis-associated mortality remains as high as $30 \%$ (1). Cardiac dysfunction is an important component of multi-organ failure induced by severe sepsis. Sepsis-induced cardiac dysfunction is characterized by ventricular dilatation, reduction in ejection fraction and reduced contractility (2). Previous evidence has shown that mitochondrial dysfunction, including impaired metabolism, altered energy generation and elevated production of reactive oxygen species (ROS), has a crucial role in the development of sepsis-induced cardiac dysfunction $(3,4)$.

The underlying mechanisms of sepsis-induced myocardial dysfunction include inflammation and oxidation reaction. Various metabolic changes have previously been detected in cardiomyocytes during sepsis and septic shock $(5,6)$. The mitochondrial enzyme aldehyde dehydrogenase (ALDH2) is crucially involved in the metabolism of acetaldehyde and other toxic aldehydes, and is essential for protecting the heart from ischemic injury (7). ALDH2 activation is sufficient to induce cardioprotection. A previous study demonstrated that treatment with Alda-1, a small-molecule activator of ALDH2, increased ALDH2 activity by two-fold and reduced infarct size by $60 \%$ in a rat in vivo model of myocardial infarction, most likely through its inhibitory effects on the formation of cytotoxic aldehydes (8). In diabetic rats, significant reduction in the left ventricular (LV) ejection fraction and fractional shortening, is accompanied by decreases in ALDH2 activity and expression (9). Transgenic overexpression of ALDH2 has been shown to effectively antagonize chronic alcohol intake-elicited myocardial hypertrophy and contractile defects $(10,11)$. Activation of ALDH2 may also mimic the cardioprotective anti-renin-angiotensin system effects of ischemic preconditioning (12).

Lipopolysaccharide (LPS) has previously been identified as a causative agent in myocardial depression during sepsis (13). There are numerous candidate pathophysiological mechanisms for the induction of myocardial dysfunction 
in sepsis. In a septic chimeric murine model, wild-type mice were shown to have decreased cardiac functions and increased myocardial tumor necrosis factor- $\alpha$ and interleukin-6 levels whereas Toll-like receptor (TLR)-4 knockout mice had attenuated responses to an LPS challenge. These previous findings contribute to the increasing evidence that TLR-4 has a role in the myocardial inflammatory response to LPS (14).

Considering that protection of cardiac function in sepsis is a serious and urgent problem, the present study aimed to explore the role of ALDH2 in LPS-induced cardiac dysfunction.

\section{Materials and methods}

Animals. Male Sprague Dawley rats (230-250 g) were purchased from the Experimental Animal Center of Zhejiang University (Zhejiang, China) and were cared for in compliance with the National Institutes of Health (NIH) Guide for the Care and Use of Laboratory Animals (NIH, Bethesda, MA, USA). All experimental protocols were approved by the Ethics Committee on Animal Experimentation of Zhejiang University (Hangzhou, China).

Materials. Aminoguanidine (AG), LPS (from Escherichia coli 055:B5), and atractyloside (Atra) were all purchased from Sigma-Aldrich (St. Louis, MO, USA). Alda-1 was obtained from Tocris Bioscience (Bristol, UK). ALDH2, prohibitin, GAPDH, and horseradish peroxidase conjugated secondary antibodies were purchased from Santa Cruz Biotechnology, Inc. (Dallas, TX, USA).

Experimental groups and survival study. The rats were randomly divided into the following groups: (1) Control group, (2) LPS group, (3) Alda-1 group, (4) LPS + Alda-1 group, (5) LPS + Alda-1 + Atra group, (6) LPS + AG group. The rats in the control group were injected intraperitoneally (i.p.) with $0.9 \% \mathrm{NaCl}$. The rats in the LPS group received a single i.p. injection of 5,10 , or $20 \mathrm{mg} / \mathrm{kg}$ LPS. The rats in the Alda-1 group received $10 \mathrm{mg} / \mathrm{kg}$ ALDH2 activator Alda-1 (i.p.). In the LPS + Alda-1 group or LPS + AG group, the Alda-1 $(10 \mathrm{mg} / \mathrm{kg})$ or iNOS selective inhibitor AG $(50 \mathrm{mg} / \mathrm{kg}$ i.p.) were injected 15 min following the LPS infusion. In the LPS + Alda-1 + Atra group, the Alda-1 and MPTP opener Atra $(25 \mathrm{mg} / \mathrm{kg}$ i.p.) was injected $15 \mathrm{~min}$ following infusion with LPS. The survival rate was recorded every $6 \mathrm{~h}$ on the first day, and then every $12 \mathrm{~h}$ for the subsequent four days.

Echocardiographic assessment. The rats were anesthetized by i.p. injection of $60 \mathrm{mg} / \mathrm{kg}$ sodium pentobarbital. An echocardiographic examination was performed using a 2-D guided M-mode echocardiograph with an $8-\mathrm{MHz}$ sector-array probe (Sonos 5500; Agilent Technologies Inc., Santa Clara, CA, USA). LV end-diastolic diameter (EDD) and end-systolic diameter (ESD) were recorded from the M-mode images. Stroke volume (SV) was equal to (end-diastolic volume - end-systolic volume), using the Teichholz correction, where end-diastolic volume $=7 /(2.4+E D D) \times E D D D^{3}$ and end-systolic volume $=7 /(2.4+\mathrm{ESD}) \times \mathrm{ESD}^{3}$. Cardiac output was calculated using the following formla: $\mathrm{SV} x$ heart rate.
All echocardiography was performed by the same qualified technician, and the data were averaged from $\geq 3$ cardiac cycles. The investigators performing and reading the echocardiogram were blinded to the treatment.

Isolation of cardiac mitochondria. The rats were euthanized by i.p. injection of $60 \mathrm{mg} / \mathrm{kg}$ sodium pentobarbital at different time points $(4,6,8,10$ or $12 \mathrm{~h}$ ) after injection with LPS, and then thoracic cavity was opened the heart was removed. The rat ventricular myocardium was excised into small pieces in ice-cold isolation buffer containing: $50 \mathrm{mmol} / 1$ sucrose, $200 \mathrm{mmol} / 1 \mathrm{mannitol}, 5 \mathrm{mmol} / 1 \mathrm{KH}_{2} \mathrm{PO}_{4}, 1 \mathrm{mmol} / 1$ EGTA, $5 \mathrm{mmol} / 13$-(N-morpholino) propanesulfonic acid (MOPS), and $0.1 \%$ bovine serum album (pH 7.4 adjusted with $\mathrm{KOH}$ ). The samples were homogenized using an Ultra Turrax homogenizer (IKA, Staufen, Germany) six times for $10 \mathrm{sec}$ at $12,000 \mathrm{rpm}$, and centrifuged at $1,000 \mathrm{x} \mathrm{g}$, for $10 \mathrm{~min}$. The supernatants, containing the mitochondrial fraction, were further centrifuged at $10,000 \mathrm{x}$ g, for $20 \mathrm{~min}$. The pellets were resuspended in isolation buffer (without EGTA) and centrifuged at $10,000 \mathrm{x} \mathrm{g}$, for a further $20 \mathrm{~min}$. The pellets were then further resuspended in isolation buffer (without EGTA). The mitochondrial purity was assessed by western blot analysis with the mitochondrial marker prohibitin and the cytosolic marker GAPDH. The protein concentration was determined using a bicinchoninic acid protein assay (Beyotime Institute of Biotechnology, Haimen, China).

Western blot analysis. Aliquots of the cardiac mitochondria, containing $20 \mu \mathrm{g}$ of protein, were separated by $10 \%$ SDS-PAGE, and transferred onto a nitrocellulose membrane (Invitrogen Life Technologies, Carlsbad, CA, USA). The membrane was incubated with the ALDH2 antibody (1:1,000 dilution) overnight, at $4^{\circ} \mathrm{C}$. Following thorough washing, the membrane was incubated with a horseradish peroxidase-conjugated secondary antibody (1:1,000 dilution) for $1 \mathrm{~h}$ at room temperature. An Enhanced Chemiluminescence kit (GE Healthcare Life Sciences, Chalfont, UK) was used to visualize the target protein. The membrane was stripped and reprobed with the prohibitin antibody for the internal loading controls. The band density was determined using Quantity One software (Bio-Rad Laboratories, Inc., Hercules, CA) and normalized to that of prohibitin.

Measurement of ALDH2 activity. The activity of mitochondrial ALDH2 was measured in $33 \mathrm{~mm}$ sodium pyrophosphate containing $0.8 \mathrm{~mm} \mathrm{NAD}^{+}, 15 \mu \mathrm{M}$ propionaldehyde and $0.1 \mathrm{ml}$ mitochondrial protein extract. Propionaldehyde, the substrate of ALDH2, was oxidized in propionic acid, while NAD+ was reduced to NADH to estimate ALDH2 activity. NADH was determined spectrophotometrically using a Tecan Infinite M200 (Tecan Group Ltd., Mannedorf, Switzerland), at an absorbance wavelength of $340 \mathrm{~nm}$. ALDH2 activity was expressed as nmol NADH/min/mg protein.

Determination of nitric oxide (NO)content and iNOS activity. The tissue concentration of NO (total nitrate and nitrite) and iNOS activity were measured using NO and NOS activity kits (Nanjing Jiancheng Bioengineering Institute, Nanjing, China), according to the manufacturer's instructions. The 
A

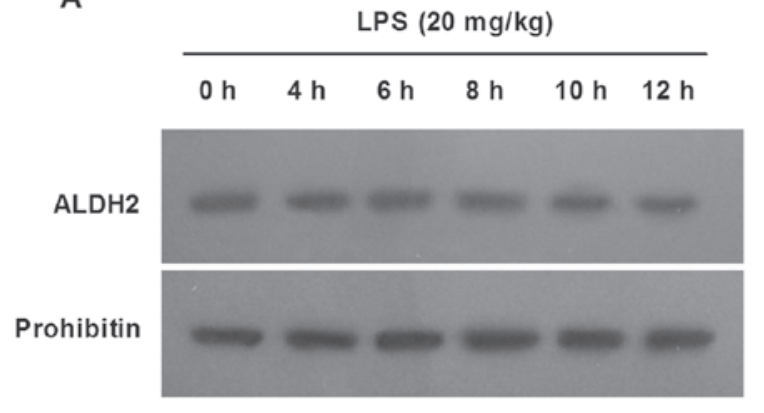

C

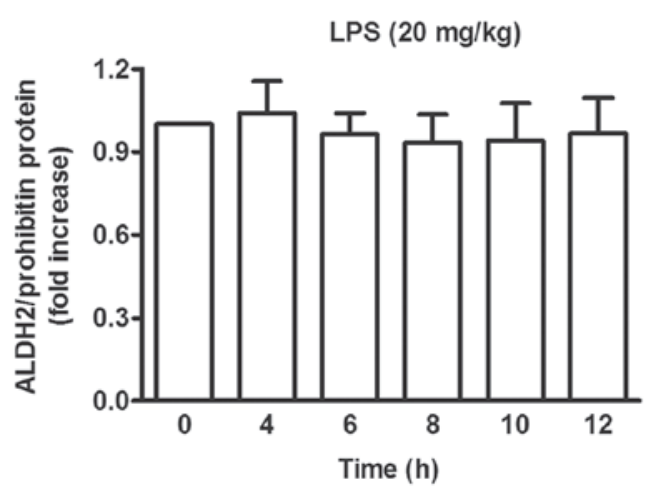

E

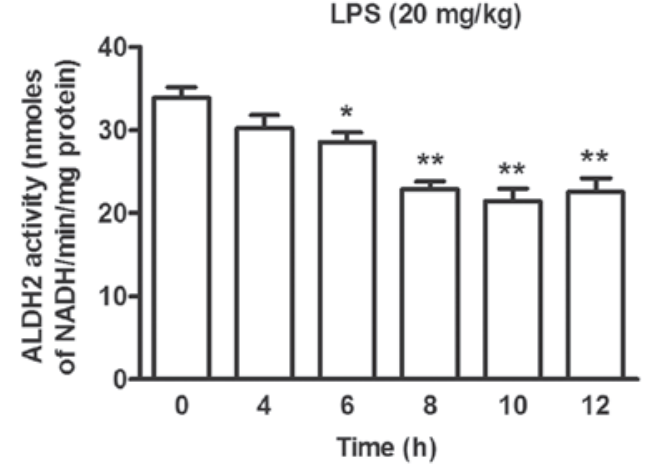

B

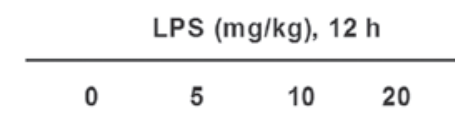

ALDH2

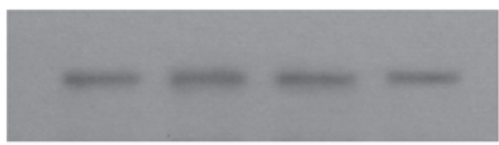

Prohibitin

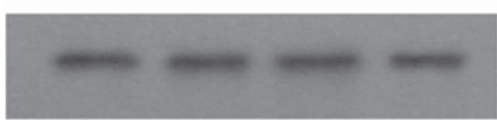

D

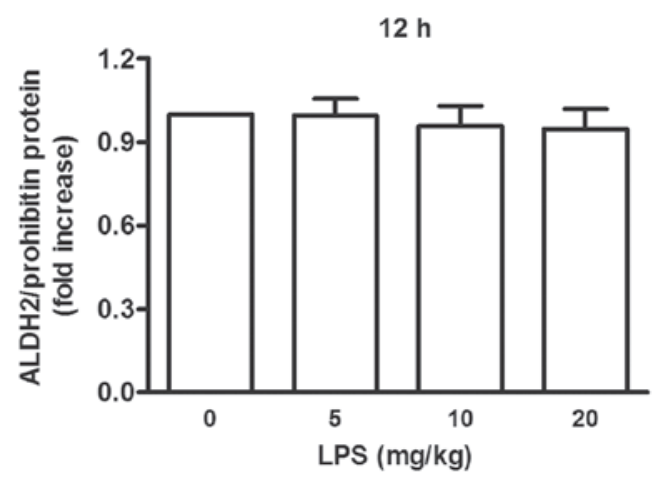

$\mathbf{F}$

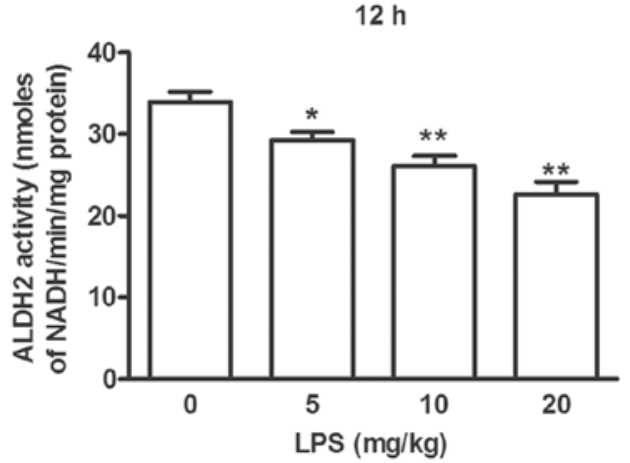

Figure 1. Aldehyde dehydrogenase (ALDH2) protein expression levels and activity in rat myocardium, following an injection of lipopolysaccharide (LPS). (A and B) Representative immunoblots obtained with ALDH2 and prohibitin antibodies, as determined by western blotting. (C and D) Densitometric analysis showing the expression levels of ALDH2 protein. The data are expressed as the means \pm standard error of the mean $(n=3)$, and as a fold increase relative to the value of the control group. (E and F) Measurement of ALDH2 activity in LPS-treated rat hearts. The data are expressed as the means \pm standard error of the mean $(\mathrm{n}=8) .{ }^{*} \mathrm{P}<0.05 ;{ }^{* *} \mathrm{P}<0.01$ vs the control group. $\mathrm{H}$, hours.

Coomassie Brilliant Blue method (Beyotime Institute of Biotechnology) was used to measure the protein concentrations.

Measurement of MPTP opening. Opening of the MPTP was determined by $\mathrm{Ca}^{2+}$-induced swelling of the mitochondria, which was measured as a reduction in A520. For measurement of the MPTP opening, mitochondria were suspended in freshly prepared swelling buffer $(120 \mathrm{mmol} / 1 \mathrm{KCl}$, $10 \mathrm{mmol} / \mathrm{l}$ Tris- $\mathrm{HCl}, 20 \mathrm{mmol} / \mathrm{l} \mathrm{MOPS}, 5 \mathrm{mmol} / 1 \mathrm{KH}_{2} \mathrm{PO}_{4}$, $\mathrm{pH} 7.4$ ) at a final concentration of $0.25 \mathrm{mg} / \mathrm{ml}$. Absorbance was measured spectrophotometrically at $520 \mathrm{~nm}$ as $\mathrm{A} 520_{\max }$. Pore opening was monitored as the decrease in absorbance at $520 \mathrm{~nm}$, which was induced by addition of $\mathrm{CaCl}_{2}(200 \mu \mathrm{mol} / \mathrm{l})$. The opening of the MPTP was indicated as a change between $\mathrm{A} 520_{\text {max }}$ and $\mathrm{A} 520_{\text {min }}(15)$.
Statistical analyses. The data are expressed as the means \pm standard error of the mean and were analyzed using Prism 5.0 (GraphPad Software, Inc., La Jolla, CA, USA) software, by a one-way analysis of variance with a Newman-Keuls' post hoc test as required. A $\mathrm{P}<0.05$ was considered to indicate a statistically significant difference.

\section{Results}

Mitochondrial ALDH2 activity decreases following treatment with LPS. The protein expression levels of ALDH2 remained unchanged following exposure to LPS $(20 \mathrm{mg} / \mathrm{kg})$; however, the activity of ALDH2 decreased after $6 \mathrm{~h}$ following LPS treatment. The ALDH2 activity decreased in a dose-dependent manner following treatment with various 
A
control
B LPS $(20 \mathrm{mg} / \mathrm{kg}, 12 \mathrm{~h})$
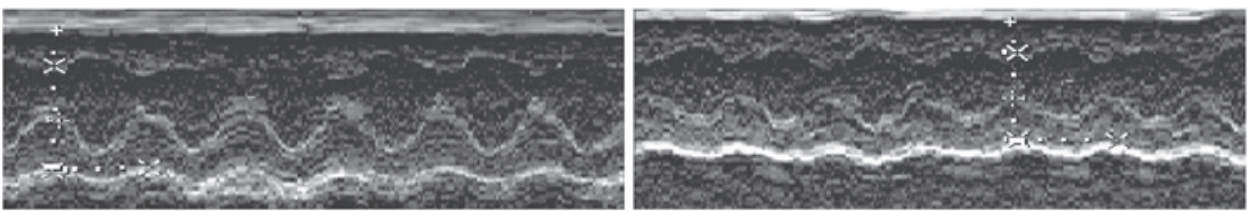

C

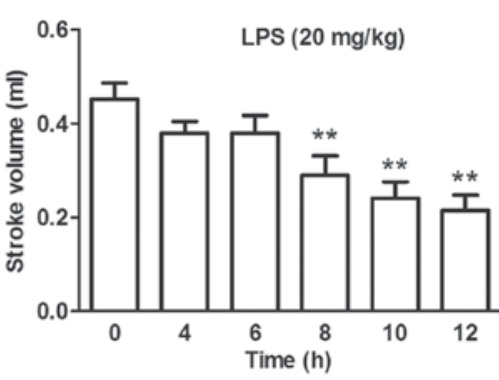

$\mathbf{F}$

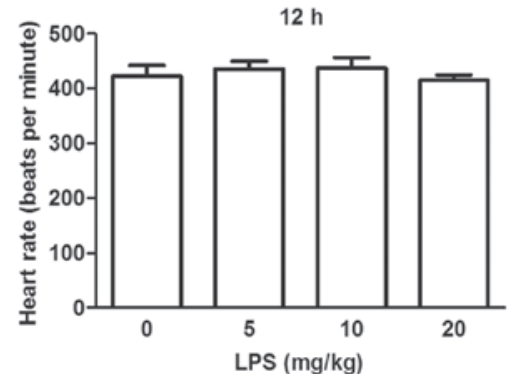

D

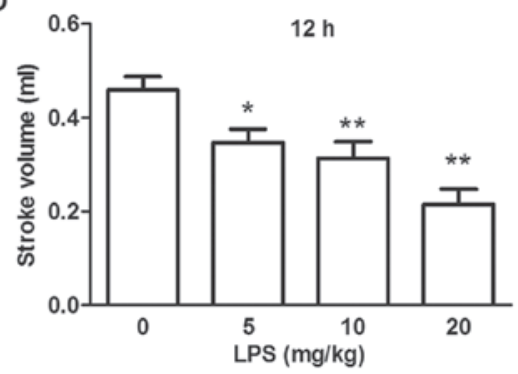

G

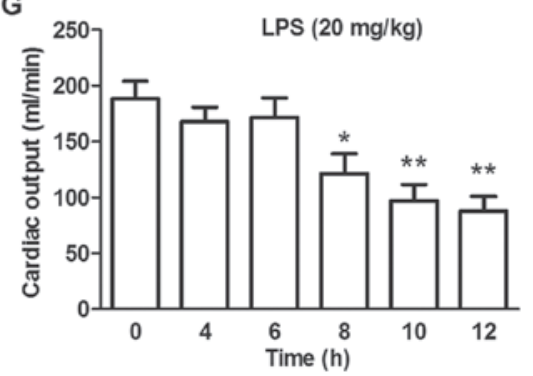

E

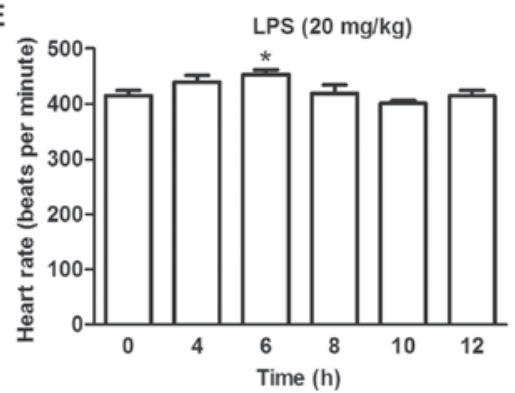

$\mathrm{H}$

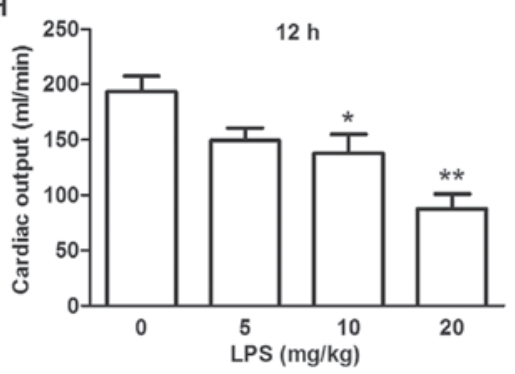

Figure 2. Cardiac function in rat hearts following an injection of lipopolysaccharide (LPS). (A and B) Representative echocardiographs of the control and LPS groups. (C and D) Measurement of stroke volume, $(\mathrm{E}$ and $\mathrm{F})$ heart rate, and $(\mathrm{G}$ and $\mathrm{H})$ cardiac output in LPS-treated rat hearts. The data are expressed as the means \pm standard error of the mean $(n=8) .{ }^{*} \mathrm{P}<0.05 ;{ }^{* *} \mathrm{P}<0.01$ vs the control group. $\mathrm{H}$, hours.
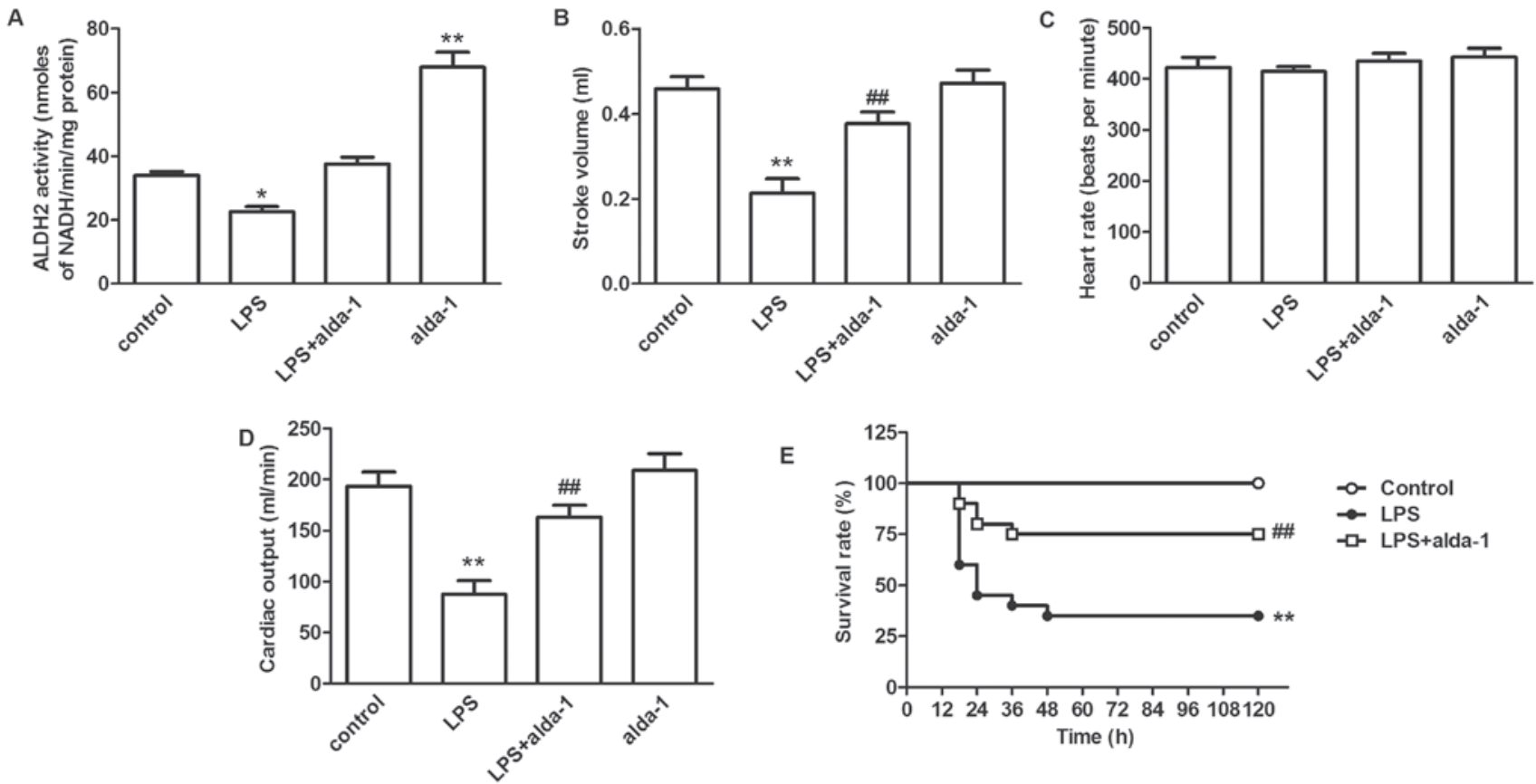

Figure 3. Effects of Alda-1 on (A) cardiac aldehyde dehydrogenase (ALDH2) activity, (B-D) cardiac function and (E) survival in lipopolysaccharide (LPS)-treated rats. (A-D) The data are expressed as the means \pm standard error of the mean $(n=8)$. (E) The data are calculated from 20 rats from each group. ${ }^{*} \mathrm{P}<0.05 ;{ }^{* *} \mathrm{P}<0.01$ vs the control group; ${ }^{\# \#} \mathrm{P}<0.01$ vs the LPS group. $\mathrm{H}$, hours.

concentrations of LPS for 12 hours $(5,10$ and $20 \mathrm{mg} / \mathrm{kg}$ ), without any effect being observed on the protein expression levels (Fig. 1).
Alda-1 protects against cardiac dysfunction and LPS-induced mortality. Treatment with LPS $(20 \mathrm{mg} / \mathrm{kg})$ induced a slight increase in heart rate at $6 \mathrm{~h}$, which then returned to normal. 

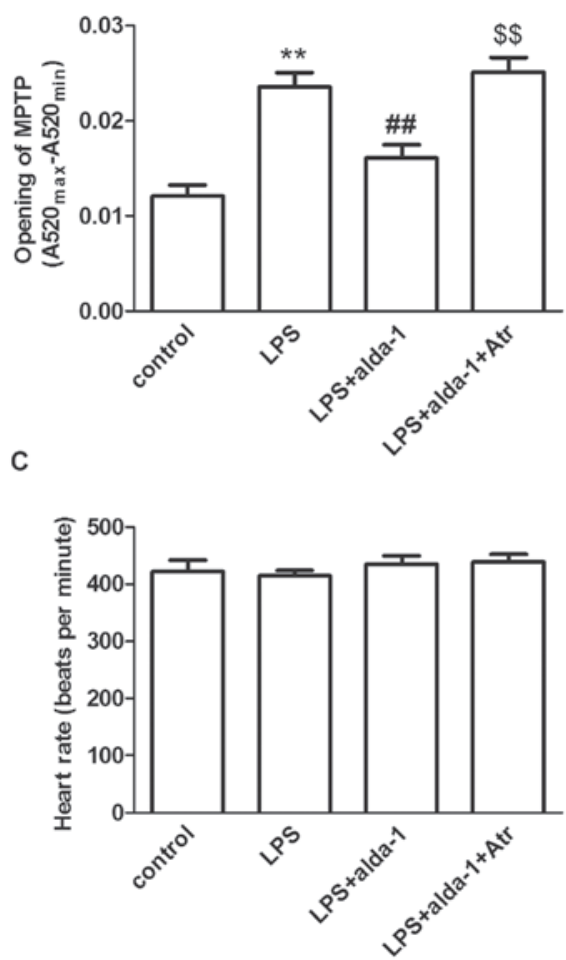

B
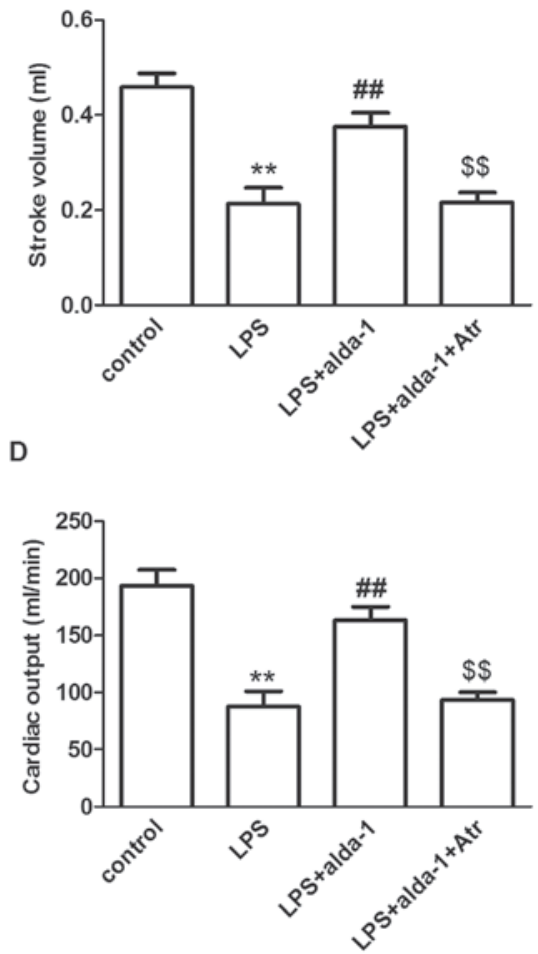

Figure 4. Effects of atractyloside (Atr) on (A) the opening of the mitochondrial permeability transition pore (MPTP), and (B-D) cardiac function in lipopolysaccharide (LPS)-treated rats. (A-D) The data are expressed as the means \pm standard error of the mean $(\mathrm{n}=8) .{ }^{* * *} \mathrm{P}<0.01 \mathrm{vs}$ control group; ${ }^{\# \#} \mathrm{P}<0.01 \mathrm{vs}$ the LPS group; ${ }^{\$} \mathrm{P}<0.01$ vs the LPS + alda-1 group.

A

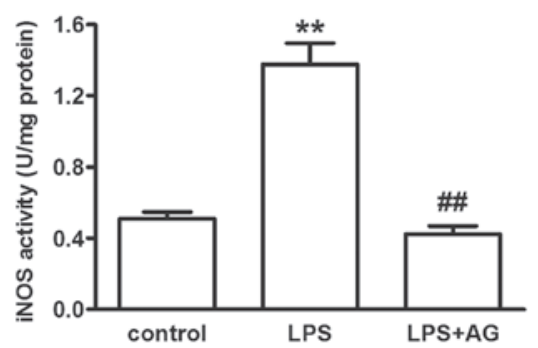

B

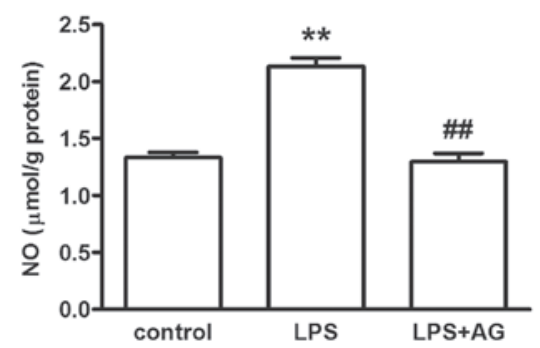

Figure 5. Effects of lipopolysaccharide (LPS) on (A) inducible nitric oxide synthase (iNOS) and (B) nitric oxide (NO) content in the rat myocardium. The data are expressed as the means \pm standard error of the mean $(n=8)$. ${ }^{* *} \mathrm{P}<0.01$ vs the control group; ${ }^{\# \#} \mathrm{P}<0.01$ vs the LPS group. AG, aminoguanidine.

Cardiac output was markedly decreased 8-12 h after LPS treatment, and both cardiac output and stroke volume decreased steadily following LPS $(5,10$ and $20 \mathrm{mg} / \mathrm{kg}$ ) treatment (Fig. 2). When Alda-1 was injected 15 min after LPS $(20 \mathrm{mg} / \mathrm{kg})$ injection, it induced an increase in ALDH2 activity, and improved cardiac function and survival rate, as compared with the controls (Fig. 3).

Alda-1 prevents LPS-induced MPTP opening. The opening of the MPTP was observed $12 \mathrm{~h}$ after LPS $(20 \mathrm{mg} / \mathrm{kg})$ treatment; however, this was prevented by treatment with Alda-1. Improvement in cardiac function in response to Alda-1 treatment was partially prevented by Atra, a selective MPTP inhibitor. These results indicate that activation of ALDH2 may be potentially beneficial for cardioprotection, through inhibition of MPTP opening (Fig. 4).
iNOS selective inhibitor AG attenuates the LPS-induced decrease in ALDH2 activity. LPS (20 mg/ $\mathrm{kg})$ induced an increase in iNOS expression and NO production, which were blocked by treatment with the iNOS selective inhibitor AG (50 mg/kg i.p.) (Fig. 5). AG additionally partially attenuated the LPS-induced decrease in ALDH2 activity and MPTP opening, and improved cardiac function (Fig. 6).

\section{Discussion}

Cardiac dysfunction is a frequent and severe complication of septic shock. There are numerous pathophysiological mechanisms associated with the induction of myocardial dysfunction in sepsis, including oxidative stress and mitochondrial dysfunction. In the present study, LPS was shown to have no effect on the protein expression levels of ALDH2; 
A

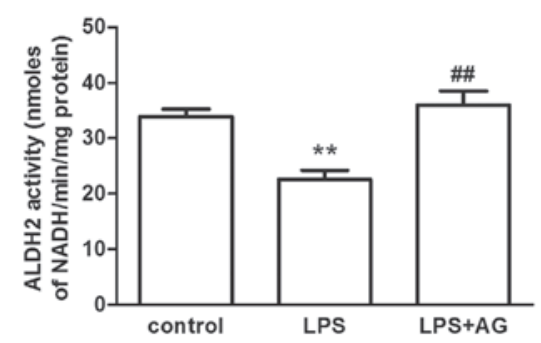

C

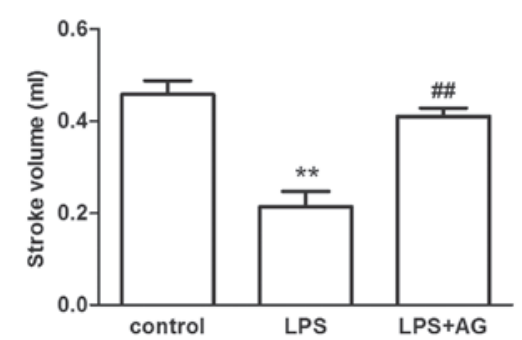

B

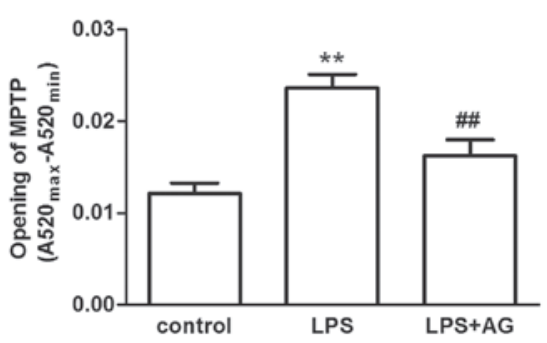

D

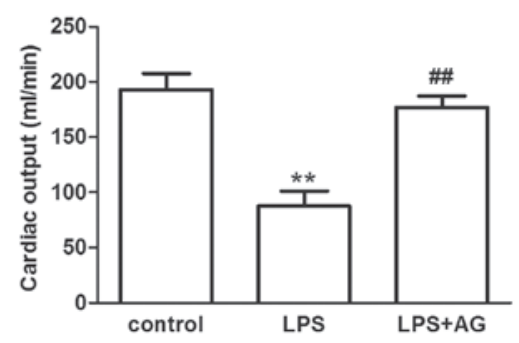

Figure 6. Effects of aminoguanidine (AG) on (A) aldehyde dehydrogenase (ALDH2) activity, (B) opening of the mitochondrial permeability transition pore (MPTP), and (C-D) cardiac function in lipopolysaccharide (LPS)-treated rats. The data are expressed as the means \pm standard error of the mean ( $\mathrm{n}=8$ ), ${ }^{* * *} \mathrm{P}<0.01$ vs the control group; ${ }^{\# \#} \mathrm{P}<0.01$ vs the LPS group.

however, it did significantly decrease ALDH2 activity, which was accompanied by cardiac dysfunction (3). LPS-induced cardiac dysfunction was reversed by Alda-1, indicating that ALDH2 activity is critical for protection against LPS-induced cardiac dysfunction.

ALDH2 is expressed in numerous tissues, with the highest levels detected in the liver, kidney, muscle, and heart (16). ALDH2 has previously been characterized as an important mediator of endogenous cytoprotection in the heart (17). ALDH2 deficiency exacerbates cardiac contractile dysfunction and promotes ER stress, following ER stress induction (18). Inhibition of ALDH2 activity has also been shown to enhance antimycin-induced rat cardiomyocyte apoptosis (19). Overexpression of ALDH2 can significantly attenuate infarct size, fractional shortening, and recovery of post-ischaemic LV function following ischemia/reperfusion injury, as well as hypoxia/reoxygenation-induced cardiomyocyte contractile dysfunction $(8,20)$. Furthermore, ALDH2 protects against diabetes-induced cardiac contraction and intracellular $\mathrm{Ca}^{2+}$ dysregulation (21).

The MPTP is opened in response to $\mathrm{Ca}^{2+}$ overload, especially when accompanied by oxidative stress, elevated phosphate concentrations and adenine nucleotide depletion $(22,23)$. Inhibition of the opening of the MPTP is considered an end target for cardioprotection $(24,25)$. Mitochondrial ALDH2 has previously been identified as a protector against oxidative stress (26). LPS-induced cardiac dysfunction is associated with an elevated production of ROS $(2,3)$. Consistent with these previous findings, in the present study a marked opening of MPTP was observed, following LPS treatment. The ALDH2 selective agonist Alda-1 prevented the LPS-induced MPTP opening. These results suggest that the cardiac protection of $\mathrm{ALDH} 2$, against an LPS-stimulated inflammatory response, may be due to inhibition of the opening of the MPTP. It is plausible that activation of ALDH2 may reduce the production of ROS, prevent MPTP opening, and alleviate cardiac myocyte injury, following exposure to LPS.

Previous reports showed that the cysteine residue of the active site in the purified yeast ALDH2 enzyme, can be modified by both NO-independent (27) and NO-dependent mechanisms (28). Expression of iNOS is increased under various inflammatory conditions, including human and experimental animal models of sepsis. In sepsis, LPS increases iNOS protein levels in the heart, liver and other tissues. iNOS generates both superoxide anions and NO, which interact to form the potent oxidant peroxynitrite. Robust NO formation from iNOS in LPS-stimulated cardiac tissue causes heart damage $(2,29)$. It has previously been reported that NO donors, such as S-nitrosoglutathione, can significantly increase nitrite concentration, while inhibiting the activity of ALDH2. NO mediates S-nitrosylation of the cysteine residue of ALDH2 and causes the reversed inhibition of ALDH2 activity (30). The present study showed, that following LPS treatment, expression of iNOS was increased, whereas ALDH2 activity was inhibited. The iNOS selective inhibitor AG partially attenuated the LPS-induced decrease in ALDH2 activity and improved cardiac function. These results suggest iNOS may have a potential role in regulation of ALDH2 activity.

In conclusion, the results of the present study suggest that ALDH2 activity may have an important role in protecting against LPS-induced cardiac dysfunction. The potential mechanisms may involve inhibition of the MPTP opening and iNOS expression. Further understanding of the beneficial role of ALDH2 in the pathogenesis of sepsis may help find a novel sepsis treatment strategy.

\section{Acknowledgements}

The present study was supported by the National Natural Science Foundation of China (no. 81272067) and the 
Zhejiang Provincial Natural Science Foundation of China (nos. Y2100082 and Y2100095).

\section{References}

1. Levy MM, Dellinger RP, Townsend SR, et al: The Surviving Sepsis Campaign: results of an international guideline-based performance improvement program targeting severe sepsis. Intensive Care Med 36: 222-231, 2010.

2. Flierl MA, Rittirsch D, Huber-Lang MS, Sarma JV and Ward PA: Molecular events in the cardiomyopathy of sepsis. Mol Med 14: 327-336, 2008

3. Flynn A, Chokkalingam Mani B and Mather PJ: Sepsis-induced cardiomyopathy: a review of pathophysiologic mechanisms. Heart Fail Rev 15: 605-611, 2010.

4. Khadour FH, Panas D, Ferdinandy P, et al: Enhanced NO and superoxide generation in dysfunctional hearts from endotoxemic rats. Am J Physiol Heart Circ Physiol 283: H1108-1115, 2002.

5. Revelly JP, Tappy L, Martinez A, et al: Lactate and glucose metabolism in severe sepsis and cardiogenic shock. Critical care medicine 33: 2235-40, 2005

6. Ramana KV, Willis MS, White MD, et al: Endotoxin-induced cardiomyopathy and systemic inflammation in mice is prevented by aldose reductase inhibition. Circulation 114:1838-46, 2006

7. Luo XJ, Liu B, Ma QL and Peng J: Mitochondrial Aldehyde Dehydrogenase, a Potential Drug Target for Protection of Heart and Brain from Ischemia/Reperfusion Injury. Current drug targets 15: 948-55, 2014

8. Chen CH, Budas GR, Churchill EN, et al: Activation of aldehyde dehydrogenase- 2 reduces ischemic damage to the heart Science 321: 1493-1495, 2008.

9. Wang J, Wang H, Hao P, et al: Inhibition of aldehyde dehydrogenase 2 by oxidative stress is associated with cardiac dysfunction in diabetic rats. Mol Med 17: 172-179, 2011.

10. Doser TA, Turdi S, Thomas DP, et al: Transgenic overexpression of aldehyde dehydrogenase- 2 rescues chronic alcohol intake-induced myocardial hypertrophy and contractile dysfunction. Circulation 119: 1941-1949, 2009.

11. Ge W, Guo R and Ren J: AMP-dependent kinase and autophagic flux are involved in aldehyde dehydrogenase-2-induced protection against cardiac toxicity of ethanol. Free Radic Biol Med 51: 1736-1748, 2011.

12. Koda K, Salazar-Rodriguez M, Corti F, et al: Aldehyde dehydrogenase activation prevents reperfusion arrhythmias by inhibiting local renin release from cardiac mast cells. Circulation 122: 771-781, 2010

13. Hickson-Bick DL, Jones C and Buja LM: Stimulation of mitochondrial biogenesis and autophagy by lipopolysaccharide in the neonatal rat cardiomyocyte protects against programmed cel death. J Mol Cell Cardiol 44: 411-418, 2008.

14. Balija TM and Lowry SF: Lipopolysaccharide and sepsis-associated myocardial dysfunction. Curr Opin Infect Dis 24: 248-253, 2011.
15. Baines CP, Song CX, Zheng YT, et al: Protein kinase Cepsilon interacts with and inhibits the permeability transition pore in cardiac mitochondria. Circ Res 92: 873-880, 2003.

16. Stewart MJ, Malek K and Crabb DW: Distribution of messenger RNAs for aldehyde dehydrogenase 1 , aldehyde dehydrogenase 2 , and aldehyde dehydrogenase 5 in human tissues. J Investig Med 44: 42-46, 1996.

17. Vasiliou V, Pappa A and Estey T: Role of human aldehyde dehydrogenases in endobiotic and xenobiotic metabolism. Drug Metab Rev 36: 279-299, 2004.

18. Liao J, Sun A, Xie Y, et al: Aldehyde dehydrogenase-2 deficiency aggravates cardiac dysfunction elicited by endoplasmic reticulum stress induction. Mol Med 18: 785-793, 2012.

19. Zhang P, Xu D, Wang S, et al: Inhibition of aldehyde dehydrogenase 2 activity enhances antimycin-induced rat cardiomyocytes apoptosis through activation of MAPK signaling pathway. Biomed Pharmacother 65: 590-593, 2011.

20. Ma H, Guo R, Yu L, Zhang Y and Ren J: Aldehyde dehydrogenase 2 (ALDH2) rescues myocardial ischaemia/reperfusion injury: role of autophagy paradox and toxic aldehyde. Eur Heart J 32: 1025-1038, 2011

21. Zhang Y, Babcock SA, Hu N, et al: Mitochondrial aldehyde dehydrogenase $(\mathrm{ALDH} 2)$ protects against streptozotocin-induced diabetic cardiomyopathy: role of GSK $3 \beta$ and mitochondrial function. BMC Med 10: 40, 2012.

22. Baines CP: The cardiac mitochondrion: nexus of stress. Annu Rev Physiol 72: 61-80, 2010.

23. Hausenloy DJ, Duchen MR and Yellon DM: Inhibiting mitochondrial permeability transition pore opening at reperfusion protects against ischaemia-reperfusion injury. Cardiovasc Res 60: 617-625, 2003

24. Hunter DR, Haworth RA and Southard JH: Relationship between configuration, function, and permeability in calcium-treated mitochondria. J Biol Chem 251: 5069-5077, 1976.

25. Halestrap AP: A pore way to die: the role of mitochondria in reperfusion injury and cardioprotection. Biochem Soc Trans 38: 841-860, 2010.

26. Ohta S, Ohsawa I, Kamino K, Ando F and Shimokata H: Mitochondrial ALDH2 deficiency as an oxidative stress. Ann NY Acad Sci 1011: 36-44, 2004

27. McDonald LJ and Moss J: Nitric oxide-independent, thiolassociated ADP-ribosylation inactivates aldehyde dehydrogenase. J Biol Chem 268: 17878-17882, 1993.

28. DeMasterEG, Redfern B, Quast BJ, Dahlseid T and Nagasawa HT: Mechanism for the inhibition of aldehyde dehydrogenase by nitric oxide. Alcohol 14: 181-189, 1997.

29. Seija M, Baccino C, Nin N, et al: Role of peroxynitrite in sepsis-induced acute kidney injury in an experimental model of sepsis in rats. Shock 38: 403-410, 2012.

30. Moon KH, Kim BJ and Song BJ: Inhibition of mitochondrial aldehyde dehydrogenase by nitric oxide-mediated S-nitrosylation. FEBS Lett 579: 6115-6120, 2005. 